\title{
Reduced Meat Consumption: from Multicriteria Argument Modelling to Agent-Based Social Simulation
}

\author{
Rallou Thomopoulos ${ }^{a^{*}}$, Nicolas Salliou $^{\mathrm{b}}$, Carolina Abreu $^{\mathrm{c}}$, Vincent Cohen $^{\mathrm{b}}$, \\ AND TimothéE FouqueraY ${ }^{\mathrm{d}}$ \\ ${ }^{a}$ IATE, University of Montpellier, INRA, Montpellier SupAgro, CIRAD, INRIA GraphIK, Montpellier, France \\ b Planning of Landscape and Urban Systems, Institute for Spatial and Landscape Development, ETH Zürich, \\ Zürich, Switzerland \\ ${ }^{\mathrm{c}}$ Computer Science Department, Institute of Exact Sciences, University of Brasília, Brasília, Brazil \\ d Écologie, Systématique, Évolution, AgroParisTech, CNRS, Paris-Sud University, University of Paris-Saclay, \\ Orsay, France \\ ${ }^{*}$ Corresponding author \\ rallou.thomopoulos@inra.fr \\ TEL: +33(0)499612217
}

Received: 7 May 2018; Published online: 18 April 2021

Invited paper from the $2^{\text {nd }}$ edition of the International School on Modeling and Simulation in Food and Bio Processes

\begin{abstract}
A second nutrition transition seems to be emerging towards more plant-based diets, curbing meat consumption in developed countries at the beginning of the $21^{\text {st }}$ century. This shift suggests that rational arguments tend to influence an increasing number of individuals to adopt vegetarian diets. This work aimed to understand and simulate the impact of different types of messages on the choice to change food diets at the individual level, and the impact of the diffusion of opinions at the collective level. It provided two results: (1) a network of arguments around vegetarian diets is modelled using an abstract argumentation approach. Each argument, formalized by a node, was connected with other arguments by arrows, thus formalizing relationships between arguments. This methodology made it possible to formalize an argument network about vegetarian diets and to identify the importance of health arguments compared to ethical or other types of arguments. This methodology also identified key arguments as a result of their high centrality in being challenged or challenging other arguments. The results of constructing this argument network suggested that any controversy surrounding vegetarian diets will be polarized around such high centrality arguments about health. Even though few ethical arguments appeared in our network, the health arguments concerning the necessity or not of animal products for humans were indirectly connected with ethical choices towards vegetarian diets; (2) an agent-based simulation of the social diffusion of opinions and practices concerning meat consumption is then introduced. The purpose of this simulation was to capture the balance of vegetarian vs. meat-based diets. It contributes to modelling consumer choices by exploring the balance between individual values and external influences such as social pressure, communication campaigns and sanitary, environmental or ethical crises.
\end{abstract}

Keywords: Sustainable food systems; Agent-based modelling; Argumentation systems; Food diet; Consumer choice criteria; Animal food product 


\section{Introduction}

The first nutrition transition involved the increase of sugar, fat, meat and processed products in human diets (Popkin, 1993) and is the dominant nutritional model today. Vranken, Avermaete, Petalios and Mathijs (2014) identified a second nutrition transition that is occurring in the most developed countries where meat consumption is currently decreasing. The transition towards the reduction of meat consumption covers a wide variety of practices ranging from occasional vegetarianism to veganism (also called "strict vegetarian") (Beardsworth \& Keil, 1991). The rationale for such a transition mainly implies ethical and health concerns (Jabs, Devine \& Sobal, 1998), but the environmental impact of meat consumption is also highlighted to a lesser degree (Ruby, 2012). McDonald (2000) conducted individual interviews with vegans and found that their nutrition transition depended on a catalytic experience that oriented individuals towards information acquisition and ultimately led to a decision to change diets. However, the information that led to the decision is not precisely known among vegetarians. Under what conditions are we likely to observe the emergence of a second transition? This paper aims to understand and simulate the impact of different types of messages on the choice to change food diets at the individual level, and the impact of the diffusion of opinions at the collective level.

As a first step, we present a methodology to explore the main arguments and the relationships between them that transitioning individuals may face. The approach is based on the principles of argumentation networks, an artificial intelligence model based on the construction and evaluation of interacting arguments. Most of the existing models are grounded in the abstract argumentation framework proposed in Dung (1995). In this framework, an argumentation system is defined by a set of arguments $\mathrm{A}$, and an attack relation between arguments R. Sets of arguments that "make sense" together are then computed and are referred to as extensions. In a subsequent study (Thomopoulos, 2018), various indicators were defined to analyze argumentation systems by highlighting consensual and non-consensual aspects of the issue debated.
As a second step, an agent-based simulation is proposed. Following the theoretical approach of Xie et al. (2011), argument networks may take advantage of being used together with agentbased modelling (ABM) to explore the emergent establishment of new social norms in the specific case of vegetarianism. Such a model can help understand the conditions under which such arguments could circulate in a population and favour the adoption of a vegetarian diet.

Agent-based models offer a way of taking inter-individual heterogeneity, social interaction and interdependence, adaptation, and decisionmaking into account at different levels. In the environmental sciences, these models have proved to be a way to capture complex characteristics of socio-ecological systems (An, 2012; Berger, 2001; Epstein \& Axtell, 1996; Filatova, Verburg, Parker \& Stannard, 2013; Matthews, Gilbert, Roach, Polhill \& Gotts, 2007; Parker, Manson, Janssen, Hoffmann \& Deadman, 2003; Rammel, Stagl \& Wilfing, 2007). In this family of ABM studies, ecology and geography are dominant (Castella, Trung \& Boissau, 2005; Grimm, 1999; Parker et al., 2003; Railsback, Lytinen \& Jackson, 2006; Verburg \& Veldkamp, 2005). However, the social sciences are also present in ABM approaches to assess and explain the complexity of human decision-making processes and behaviours (Epstein \& Axtell, 1996; Janssen, Walker, Langridge \& Abel, 2000; Janssen \& Ostrom, 2006; Robinson et al., 2007; Schelling, 1971; Simon, 1997).

In the food sciences, historically based on process-oriented studies, ABMs are absent from the range of models used to approach food quality and, as a more recent concern, food sustainability. Recent studies related to multiagent systems applied to the agri-food sector are those based on the argumentation theory (Bourguet, Thomopoulos, Mugnier \& Abecassis, 2013; Thomopoulos, Croitoru \& Tamani, 2015; Thomopoulos, Moulin \& Bedoussac, 2017). However, social issues are deeply involved in food system sustainability. Consumer demand, environmental awareness, willingness to pay, acceptability of products and the transmission of new food habits are all key factors to analyze the food system. Consequently, there is an urgent need and a real challenge to take food-related social 
behaviors into account and to integrate them into food policy analysis.

A previous approach for combining argumentation and simulation for decision support in food systems was proposed in Thomopoulos et al. (2017). The simulation model considered in that study was systems dynamics. In this paper, an ABM simulation of the social diffusion of opinions and practices concerning meat consumption is proposed. It aims to capture the balance of vegetarian vs. meat food diets. More specifically, we aim to understand the balance between individual values and external influences such as social pressure, communication campaigns and sanitary, environmental or ethical crises in the decision-making of individuals with regard to their food diet.

\section{Materials and Methods}

\subsection{Argumentation Formalism}

In order to model arguments involved in vegetarian transitions, we used an abstract argumentation approach (Dung, 1995; Rahwan \& Simari, 2009; Thomopoulos, 2018). An argumentation system is usually represented as an oriented graph where nodes are arguments and edges are attack relations between arguments (Figure 1). Considering Dung (1995) seminal work on argumentation, an argument and the attack relation are abstract and can be instantiated and defined in different ways in different contexts (Walton \& Macagno, 2015). Dung himself stated: "an argument is an abstract entity whose role is solely determined by its relations to other arguments. No special attention is paid to the internal structure of the arguments." For example, an argument can be a set of statements composed of a conclusion and at least one premise, linked by an inference or a logical relationship. Attacking an argument can be achieved in different ways:

1. by raising doubts about its acceptability through critical questions;

2. by questioning its premises;

3. by suggesting that the premises are not relevant to the conclusion; or
4. by presenting an argument with an opposing conclusion.

In all these cases, an attack relation is said to exist.

Even though Dung's framework is theoretically sound, it is not straightforward enough to be applied to real-life situations. Indeed, one of the initial difficulties is how to define an argument in order to properly reflect stakeholders' statements in a debate. Unfortunately, there is still no general model that can be used to formalize a natural argument (i.e., an argument stated by a stakeholder during a discussion in natural language) and input in an abstract argumentation framework in a real decision-making context. Quoting Baroni and Giacomin (2009): "While the word 'argument' may recall several intuitive meanings, like the ones of 'line of reasoning leading from some premise to a conclusion' or of 'utterance in a dispute', abstract argument systems are not (even implicitly or indirectly) bound to any of them: an abstract argument is not assumed to have any specific structure but, roughly speaking, an argument is anything that may attack or be attacked by another argument." Indeed, the structure of an abstract argument does not correspond to the intuitive understanding of what an argument is. Moreover, the notion of "attack between arguments" does not have a natural and direct correspondence to practical expressions used by stakeholders when debating. Finally, representing arguments as an oriented graph can be a difficult task for stakeholders: when the number of arguments and/or attacks is large, the graph becomes illegible and difficult to interpret by the stakeholders. For all these reasons, the ways that arguments and attacks are identified and modelled is part of the project contributions. They are thus presented in Section 3.

\section{The arguments}

In our project, we needed to find a practical way of defining arguments that are used in the process of decision-making. In such a context, arguments can be intuitively thought of as being statements to support, contradict or explain opinions or decisions (Amgoud \& Prade, 2009). More precisely, 


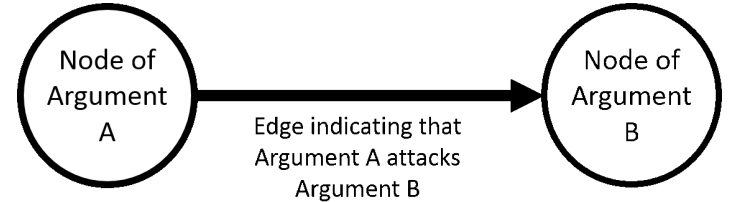

Figure 1: General graphical representation of an argumentation system

in decisional argumentation frameworks (Ouerdane, Maudet \& Tsoukiàs, 2010), the argument definition is enriched with additional features, namely the decision (also referred to as 'action', 'option' or 'alternative') and the goal (also referred to as 'target'). In other studies, arguments are also associated with specific actors. An application of a decision-oriented argumentation framework to a real-life problem concerning food policy can be found in Bourguet et al. (2013), where a recommendation regarding the provision of whole-grain bread was analyzed a posteriori. In this case, each argument is associated with the action it supports.

\section{The attack relation}

Now, let us consider the attack relation. In structured argumentation (i.e., logic-based argumentation frameworks where arguments are obtained as instantiations over an inconsistent knowledge base) three kinds of attacks have been defined: undercut, rebut and undermine (Besnard \& Hunter, 2008). The intuition of these attack relations is either to counter the premise of the opposing argument ('undercut'), the conclusion ('rebut') or to attack the logical steps that allowed the inference between the argument's premise and conclusion ('undermine'). In abstract argumentation, the set of attacks is simply considered as provided a priori. Another possibility that can be considered is to enhance the argumentation framework with a set of preferences expressed, for instance, as weights representing uncertainty. In our project, we needed to choose a practical way to define the attack relation.

\section{$2.2 \quad$ Agent-Based Modeling}

Agent-based models are computational models used to simulate the actions and interactions of individual or collective autonomous agents in order to assess their effects on the system as a whole. They attempt to reproduce and predict the emergence of complex phenomena induced from the micro-scale to the macro-scale. Their principle is that simple local behavioural rules generate complex global behaviour. An overview of their early history can be found in Samuelson (2000), and more recent developments in Samuelson and Macal (2006). The model introduced was built and run on two different agent-based modelling and simulation platforms, namely CORMAS and GAMA (Taillandier et al., 2018). The CORMAS platform (http://cormas. cirad.fr/) was specifically developed by CIRAD - the French international cooperation organization for agricultural research in the global South - to simulate natural resource management, and is oriented towards the representation of interactions between stakeholders about resource use. In CORMAS, entities are categorized into three types: spatial entities describing the space at different aggregation levels, passive entities that are objects that can be manipulated by social agents, and social entities that can make decisions, move, and interact with other agents. The GAMA platform platform (http://gama-platform.org) was developed by a consortium of research teams using the JAVA language. It is generalist in its application domains and is particularly wellsuited for connection with geographical information system data and visualization.

\section{Results and Discussion}

\subsection{Argumentation Results}

\section{Contributions to Modelling Arguments and Attacks}

We introduced the specification of an argument as a tuple $\mathrm{a}=(\mathrm{I} ; \mathrm{T} ; \mathrm{S} ; \mathrm{R} ; \mathrm{C} ; \mathrm{A} ; \mathrm{Is} ; \mathrm{Ts})$ where:

I was the identifier of the argument; 
$\mathbf{T}$ was the type of the argument (with values in favor of, denoted by ' + ', or against, denoted by '-', the vegetarian option);

$\mathbf{S}$ was the statement of the argument, i.e., its conclusion;

$\mathbf{R}$ was the rationale underlying the argument, i.e., its hypothesis;

C was the criterion on which the argument relies;

A was the actor who proposes the argument;

Is was the information source containing the argument:

Ts was the type of source the argument comes from.

For any argument $a$, we denoted by $\mathrm{I}(a)$, $\mathrm{T}(a), \mathrm{S}(a), \mathrm{R}(a), \mathrm{C}(a), \mathrm{A}(a), \mathrm{Is}(a), \mathrm{Ts}(a)$, the identifier, the type, the statement, the rationale, the criterion, the actor, the information source and the information type of argument $a$, respectively.

Considering the reality of stakeholders' debates and our model to formalize arguments, we chose to model the attack relation in the following way. Attacking an argument $a$ was achieved by:

1 explicitly raising doubts about its acceptability by expressing a counter-argument citing $a$ or the information source containing $a$;

2 implicitly raising doubts about its acceptability by expressing a counter-argument contradicting $a$ through undercut, rebut or undermine. Formally, we considered the following attack relation:

Let $a$ and $b$ be two arguments. Then, $a$ attacks $b$ if and only if the following two conditions are satisfied:

$$
\begin{aligned}
T(a) & \neq T(b) \\
\{R(a), S(a)\} & \Rightarrow \operatorname{not}\{R(b), S(b)\}
\end{aligned}
$$

The first condition expresses the fact that one of the arguments $a$ and $b$ is in favour and the other against the vegetarian option. The second condition expresses the inconsistency of $a$ and $b$, i.e., if the hypothesis and the conclusion of argument $a$ are assumed to be true, then the hypothesis of $b$ or the conclusion of $b$, or both, do not stand.

\section{Exploring the Literature}

Following the above formalism, we extracted arguments in favour or not of reducing animal product consumption. Our sources of arguments were newspapers, grey literature and the top ten Google search words ("vegetarian diet"; "vegan diet"; "vegetalism argument"). The latter inquiry was added to the pool of popular scientific papers, WebMedia articles and blog posts. We thoroughly read each source and extracted all of the arguments as expressed by their authors. For each argument, we attributed a criterion ("Nutritional"; "Economic"; "Environmental"; "Anthropological"; "Ethical"; "Health" or "Social") and noted the source expressing this argument ("Journalist"; "Scientist", "Philosopher"; "Blogger", etc.). We consequently obtained 114 arguments.

Table 1 displays a sample of the set of arguments considered in our case study. Each argument was first formalized by an identification number, whether it was in favour or not of meat reduction diets (+/-), its main statement and rationale such as: "Vegan diet is related to vitamin B12 deficiency" (Statement) since "plants do not contain vitamin B12" (Rationale). Other information (Actor, Information source \& Type of source) characterized the origin of the argument. Based on this first step, we then formalized attacks between them. An attack occured when an argument contradicted another one. For example, the argument "1" quoted above is contradicted by the following argument " 28 - Properly planned vegetarian or vegan diets fit all stages of life" since "Nutrient needs are satisfied and growth is normal." When these arguments are graphically formalized, each one is represented as a node, and an attack is a vertex connecting both arguments where the arrow points to the direction of the attack. In our case study, we identified 155 attacks connecting 55 arguments out of a total of 114 . 
138 Thomopoulos et al.

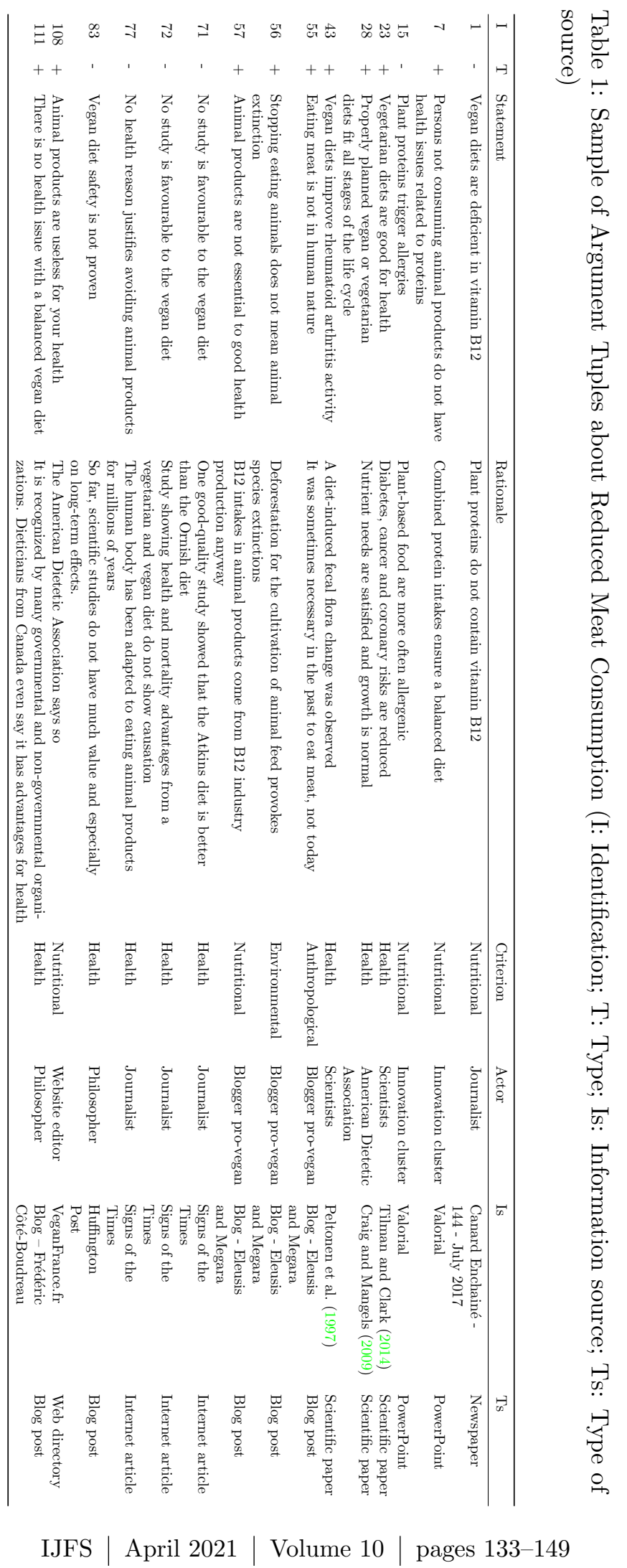




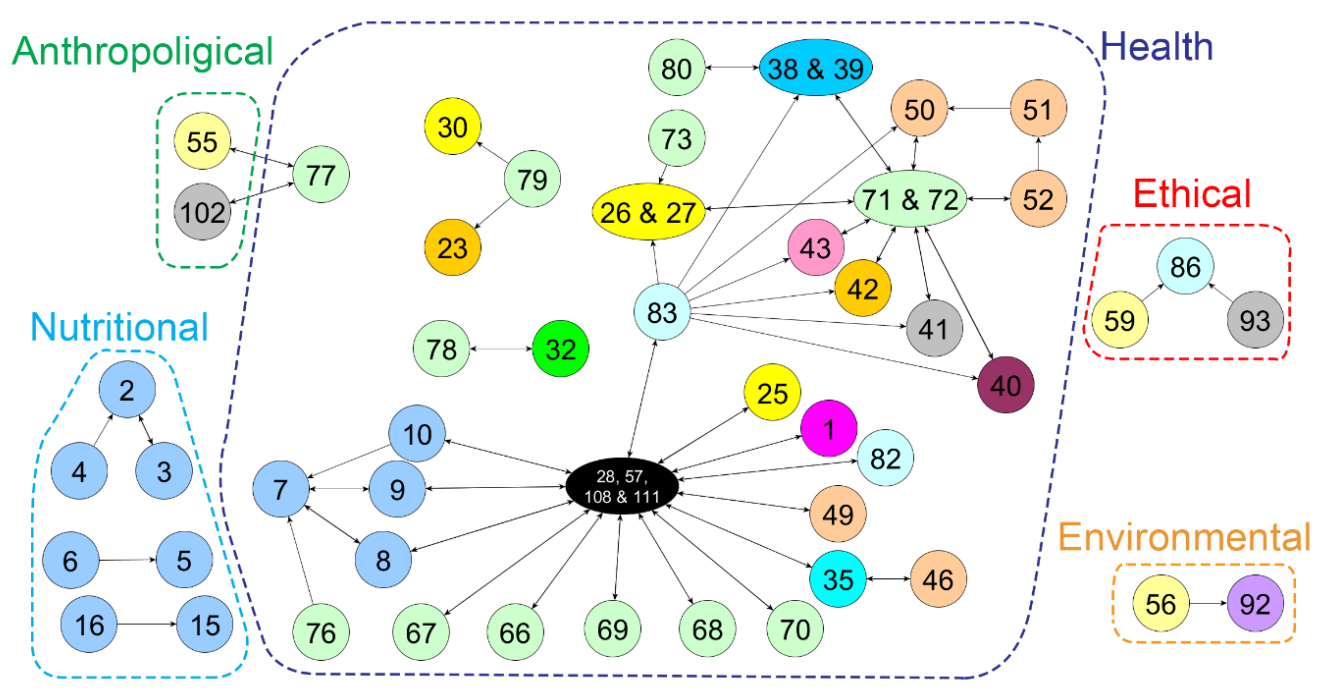

Figure 2: Graphical Representation of Arguments and Attacks about Reduced Meat Consumption

\section{Graphical representation of the argument network}

In order to make a graphical representation of the argument network, we used the visualization program Yed Graph Editor (version 3.17.1). We chose to represent only arguments that were connected with at least one attack. Hence, 55 arguments were kept and 59 were rejected. The result is displayed in Figure 2. Each number corresponds to one argument expressed by one source. Arguments were grouped in dashed lines according to the criterion they were based on, which is indicated in the legends (Nutritional, Ethical, etc.). Each argument node received a specific colour according to its source (e.g., pink for argument 1 from the newspaper "Le Canard Enchainé", No. 144, published in July 2017, blue for arguments 2 to 16 from the Valorial competitiveness cluster's presentation, etc.). For visualization purposes, we merged identical arguments in the same node when they were repeated and came from the same source.

\subsection{Agent-Based Simulation Results}

\section{Conceptual model}

\section{Scenario Description}

The system modelled was a country consisting of a population of $\mathrm{N}$ citizens. Each citizen was characterized by: (i) a constant level of need for quality regarding food (environmental preservation, ethics, health, taste, etc.); (ii) a variable level of perception of meat products corresponding to these criteria. Depending on these two levels, citizens had a behaviour regarding their food diet - either meat consumers if their perception exceeded their needs, or vegetarian if their needed exceed their perception. This principle is represented in Figure 3. Moreover, each citizen had some resistance to change, represented by the resistance rate.

Within this system, each citizen communicated with his/her direct neighbours and may then have changed his/her perception of meat consumption and, consequently, his/her diet. Citizens were also influenced by advertising campaigns that target every citizen and tend to increase meat product perceptions in the whole 


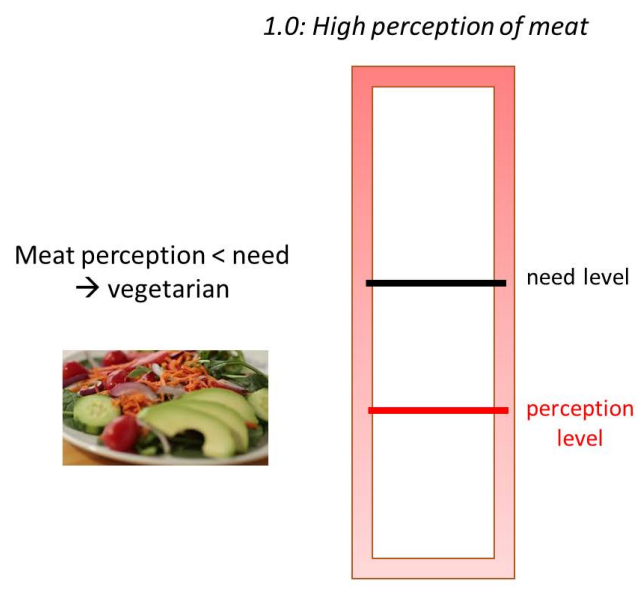

0.0: Low perception of meat

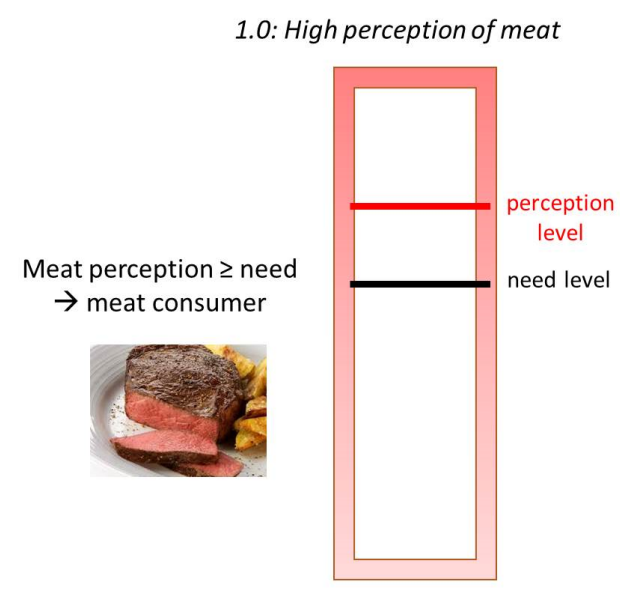

0.0: Low perception of meat

Figure 3: Behavioural model of food diet choice

country. Finally, crises broke out with a given frequency. These represented, in particular, sanitary crises and, more generally, ethical or environmental crises. All of the citizens received the information, all the more since they were spatially close to the crisis event. Their perception of meat products may have been impacted.

Initial parameters subject to stochasticity included the distribution of need and perception levels in the population with respect to some initial proportions of vegetarians and meat consumers. The output parameter observed was the evolution of food diets over time.

\section{Working Hypotheses}

The ABM developed was based on the following assumptions:

- A cognitive theory of food perceptions and needs. This was where the ABM was related to arguments in favour or not of a diet, and where the importance attached to the criteria underlying arguments for a given diet was expressed as a level of needs. The failure of a food diet to be perceived as being consistent with these needs led to a behavioural change in the ABM, which could not be captured by the argumentation system alone. In Schluter et al. (2017), six categories of human decision-making behaviours in socioecological systems are described. In the present paper, we drew on the "habitual behavior", described by the following characteristics (quoting Schluter et al. (2017)):

'Behavior is initially deliberate and goaldirected'

'if new behaviour is rewarded, the chances increase that it will be repeated'

'repeatedly obtaining satisfactory rewards reinforces the behaviour'

'the selection of behaviour will be automatic as long as needs are satisfied'

'the actor will stop automatic behaviour and deliberate about alternative behaviours if need satisfaction drops below a critical level'

'if the reward devalues or disappears, habitual behaviour persists at first, but will go extinct after longer absence of reward.'

- Three triggers for vegetarian/meat product demand:

- occurrence of sanitary, environmental 


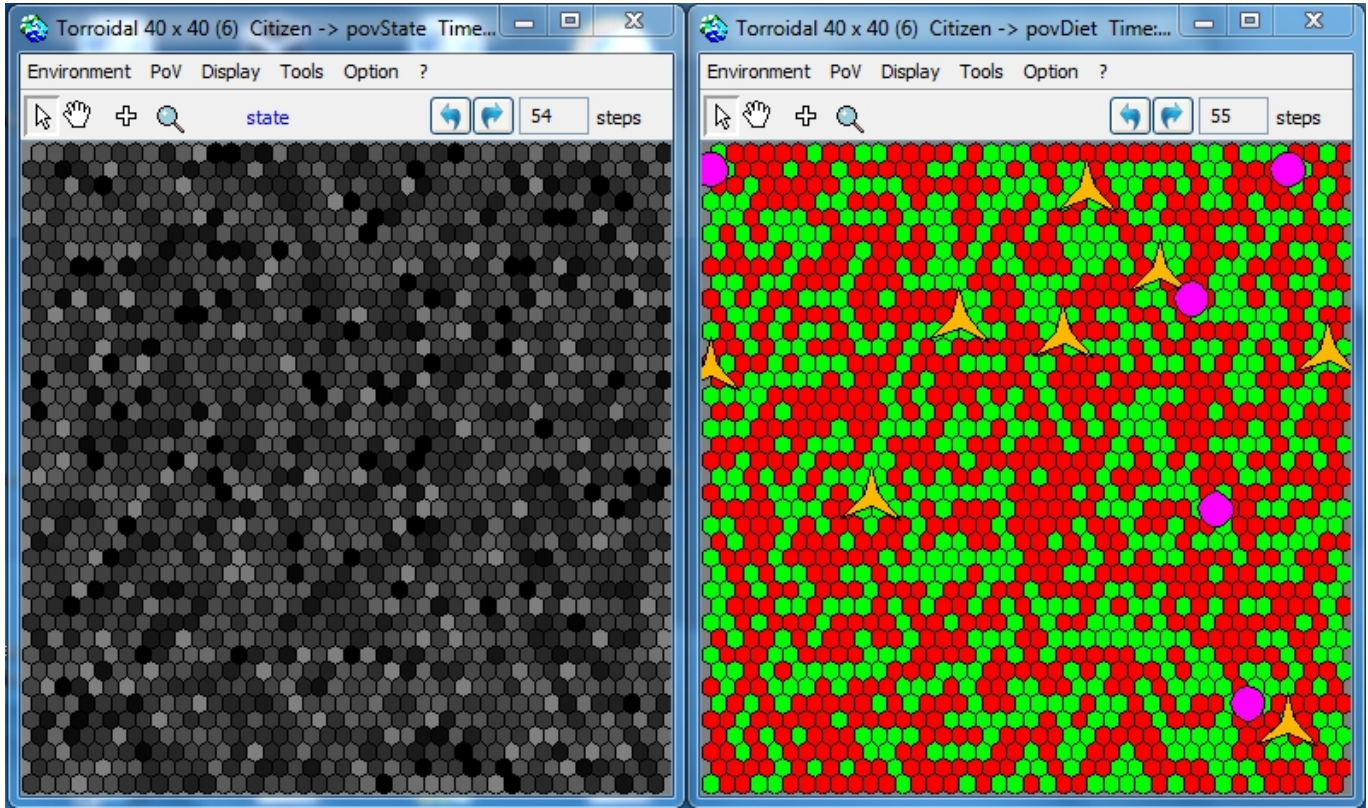

Figure 4: A running simulation in CORMAS

and ethical crises;

- neighbourhood effect;

- advertising campaigns.

The influences of these triggers were assumed to follow the opinion diffusion model (Deffuant, Amblard, Weisbuch \& Faure, 2002). An opinion (in this case, a perception of meat products) was represented by a numerical float value between 0.0 and 1.0, initialized with a random value that was less than needs for vegetarians, and greater than needs for meat consumers. At each step, agents consulted their neighbours and updated their opinions as follows. When agent $\mathrm{x}$ consulted his/her n neighbours yi (i $\epsilon[1$; n]), his/her new opinion op became:

$o p_{x}(t+1)=r R * o p_{x}(t)+(1-r R) *{\overline{o p_{y_{i}}}(t)}_{i}$

The resistance rate $r R$ was a parameter of the simulation, i.e., a number in $[0.0,1.0]$ and $\overline{o p}$ is the mean opinion.

\section{Simulations Results}

Figure 4 displays a running simulation of the model developed, implemented in the CORMAS platform. The left part shows consumer perceptions of meat products (the darker, the higher). The right side displays the resulting food diets: green for vegetarians, red for meat consumers. The yellow stars depict the occurrence of a crisis that tends to decrease people's perceptions of meat products, all the more since the crisis localization is close. The pink circles depict the occurrence of an advertising campaign that tends to increase people's perceptions of meat products. Although localized (in order to visualize them), advertising campaigns were nationwide and impacted every citizen, as already mentioned.

Figure 5 shows a running simulation of the same model, implemented in the GAMA platform. As in CORMAS, food diets are represented in green for vegetarians and in red for meat consumers. Perceptions of meat products are represented by the sizes of the circles (the larger the circle, the higher the perception). The left part of the screen makes it possible to display and easily modify simulation parameters. 
142 Thomopoulos et al.

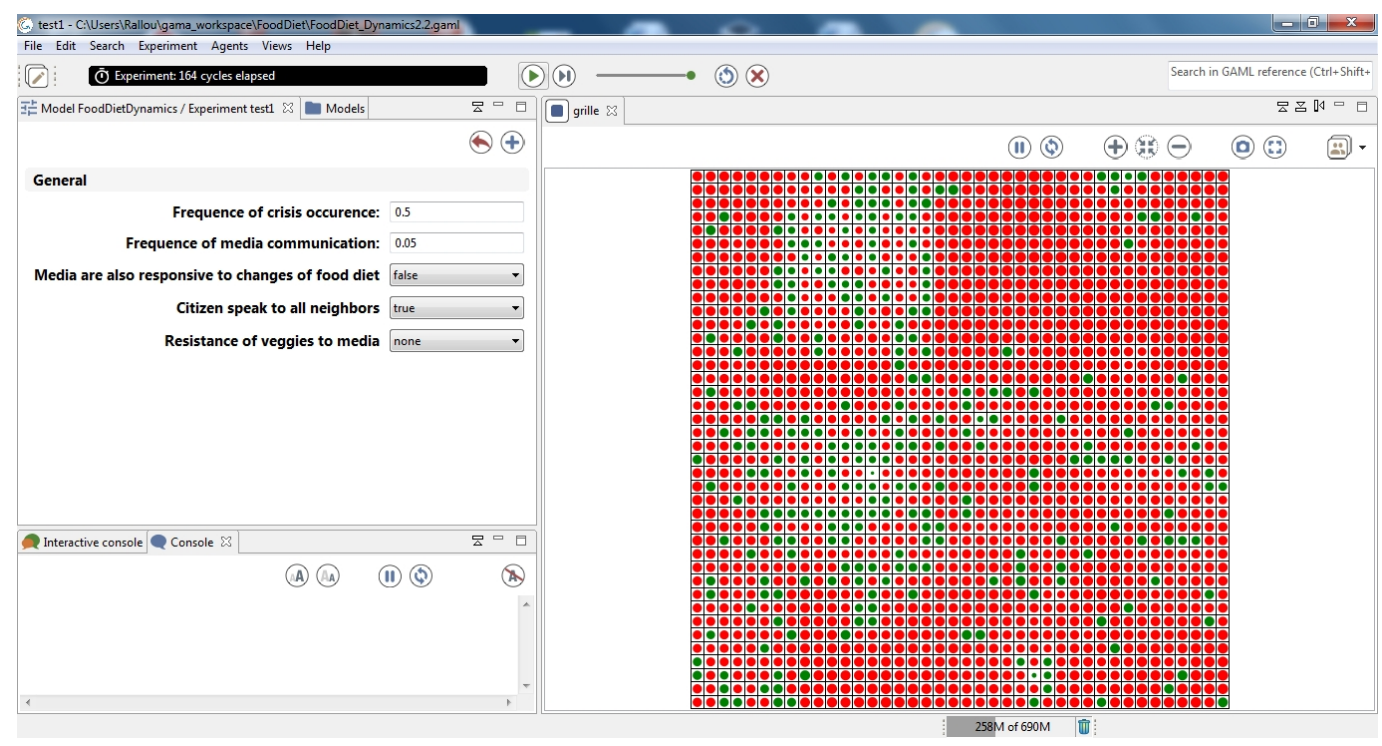

Figure 5: A running simulation in GAMA

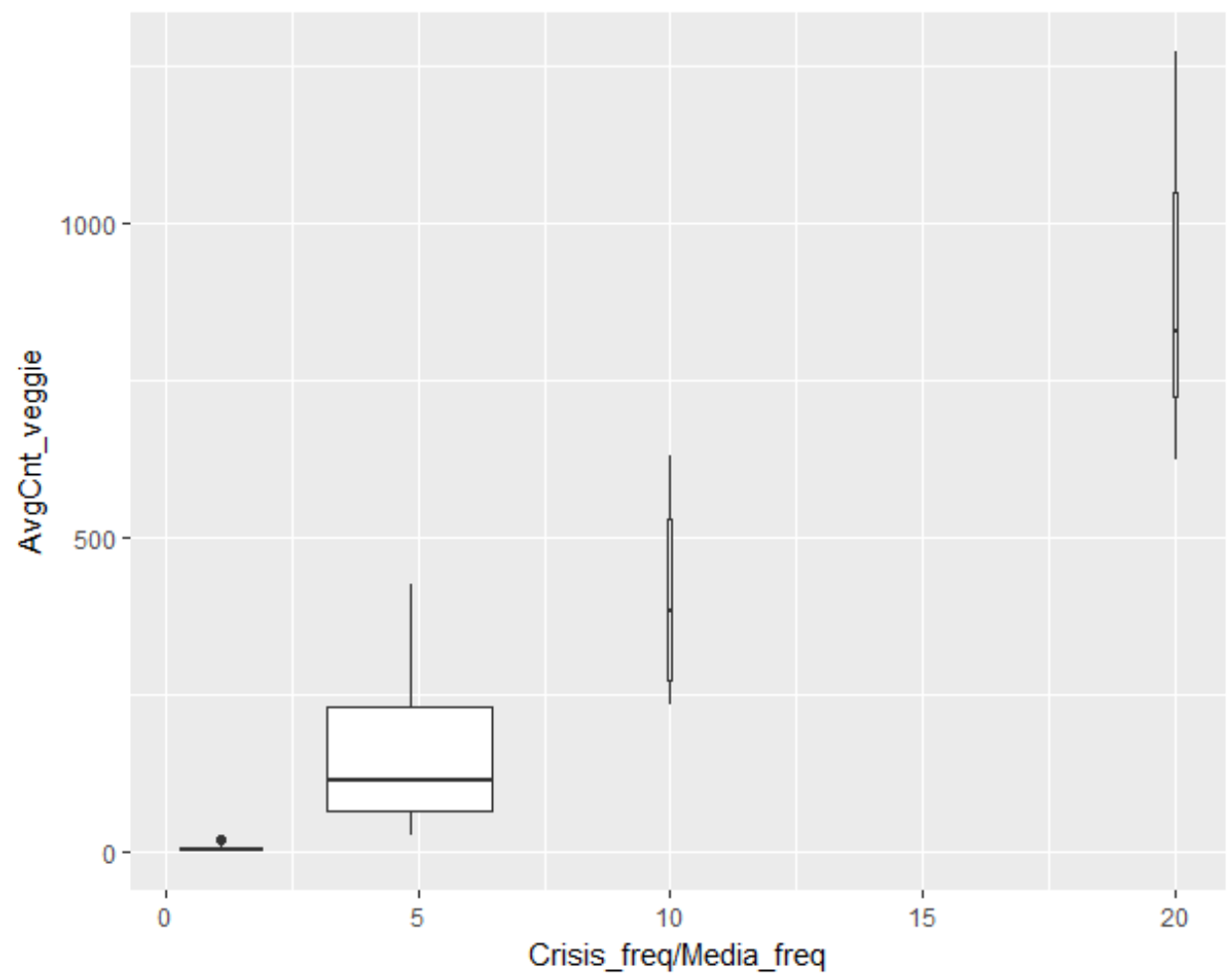

Figure 6: Effect of the ratio between the frequency of crises and that of communication campaigns 


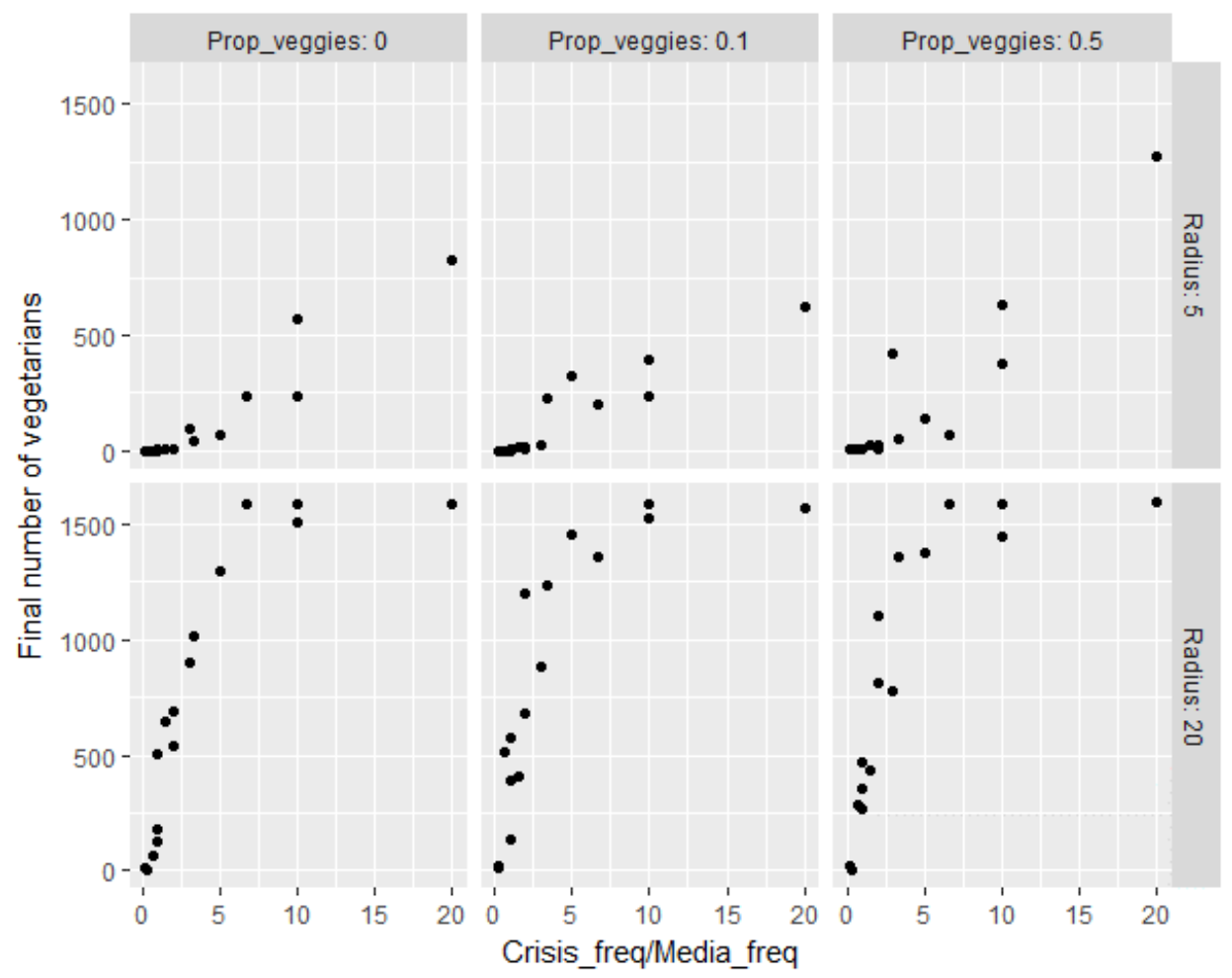

Figure 7: Combined effects of the initial proportion of vegetarians and of the radius of crises

Each step was been calibrated to correspond to about one month, so that the whole simulation corresponded to approximately 15 to 20 years.

\section{Sensitivity Analysis}

Sensitivity analysis raw results are given in Figures 6 to 9 and further commented in Section 3.3. The parameters analyzed are, respectively:

- the ratio between the frequencies of crises and communication campaigns (Figure 6);

- the initial proportion of vegetarians (Figure 7);

- the radius of crises (Figure 7);

- the progression over time of the resistance rate, constant or adaptive (Figure 8). In the latter case, the resistance rate tended to increase for individuals who had already experienced a food diet change, reflecting some "no going back" effect;

- the introduction of resistance to communication campaigns (Figure 9);

- the introduction of a selective influence of neighbours, where those with perceptions closer to the agent's perception are more carefully listened to (Figure 9).

\subsection{Discussion}

Our argument network structure revealed two main elements in particular. First, it was observed that arguments about Health were by far the majority of the arguments identified. They represented $47 \%$ of all of the 114 arguments identified and $63 \%$ of the arguments involved in at least one attack. As a matter of comparison, 
144 Thomopoulos et al.

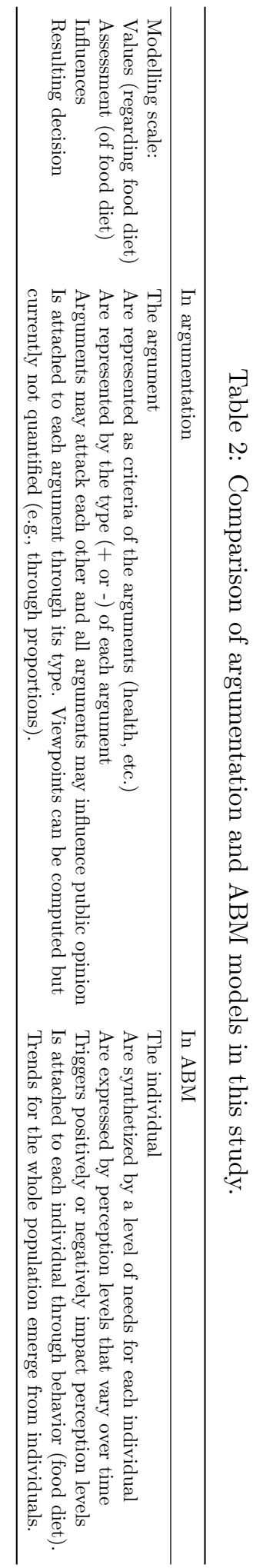

IJFS | April $2021 \mid$ Volume $10 \mid$ pages 133-149 


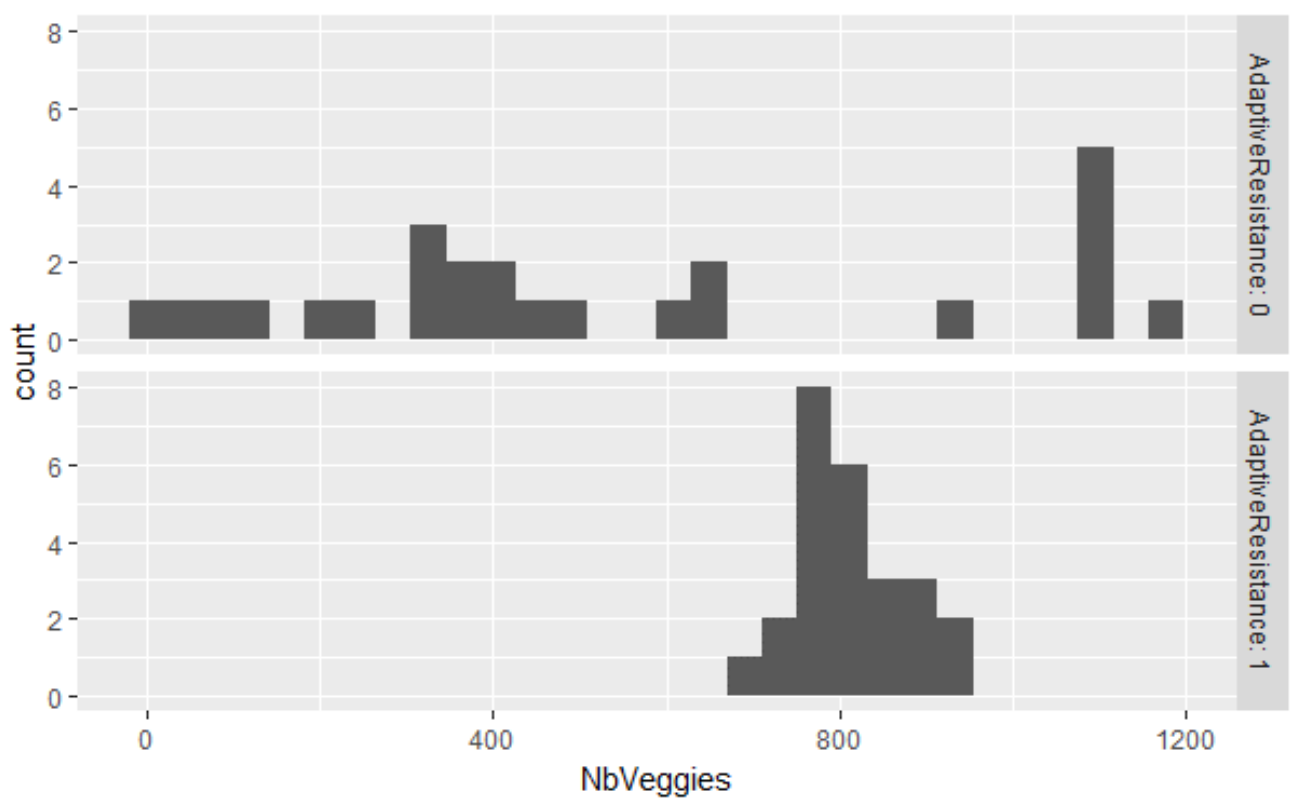

Figure 8: Effect of the adaptive resistance rate

ethical arguments represent only $3 \%$ of all arguments identified. Second, some key arguments emerged due to their centrality. Three arguments were involved in more than four attacks. The first argument, grouped under identification numbers 28, 57, 108 and 111 (the black node in Figure 2), refered to a scientific paper from the American Dietetic Association stating that "Properly planned vegetarian or vegan diets fit all stages of life" (Craig \& Mangels, 2009). The second argument, grouped under identification numbers 71 and 72, was a journalist's statement that "No study is favourable to the vegan diet". The third argument, number 83, stated that "vegan diet safety is not proven", questioning the validity of scientific studies in this regard. These arguments would probably be key arguments in potential controversies about vegetarian diets due to their generality and to their polarized nature on the question of such diet viability from a health perspective.

The major importance of health issues surrounding vegetarian diets was in line with findings in Ruby (2012) review of vegetarian studies. In contrast the importance of ethical arguments that was stressed by Ruby (2012) did not appear in this modelling. This could be explained by the more complex nature of ethical arguments as well as our choice of search words in Google that focused on diets. However, from an ethical perspective, it seemed that the health issue (whether or not vegetarian diets are healthy) was central since vegetarianism may be defended from the baseline of animal products not being necessary for human health (Francione, 2015).

In this study, we built the network and proposed a structural analysis. Abstract argumentation led to further analysis and, in particular, the rejection of attacked arguments without any argument to defend them. Such analyses allow for new indicators such as those that measure the degree of controversy based on rejected argument ratios (Thomopoulos, 2018; Thomopoulos et al. 2017), which can better identify potential controversies.

The present work illustrates a promising coupling of the argumentation theory and agent-based modeling. The use of ABM is complementary since it allows for the exploration of conditions under which arguments could spread in a pop- 

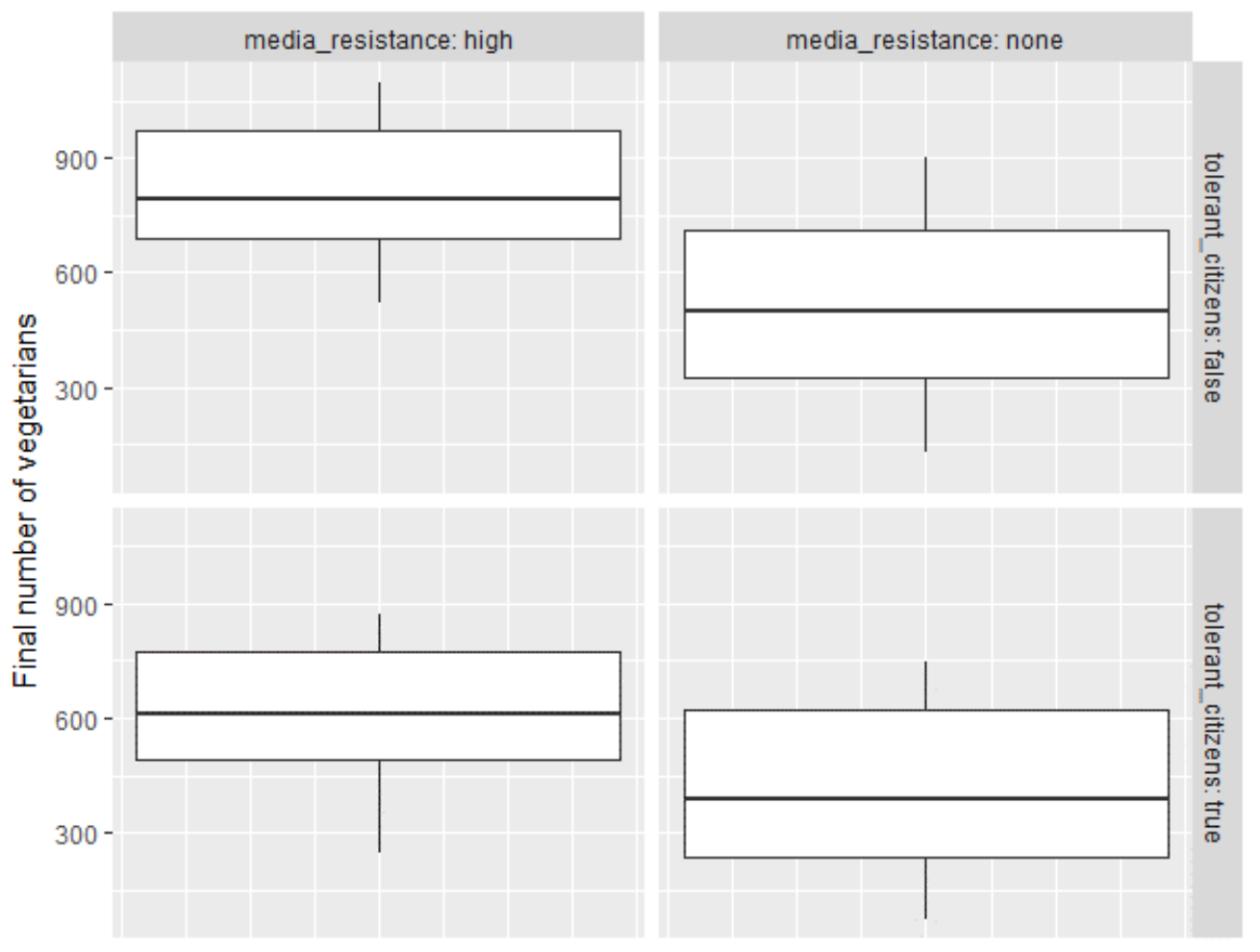

Figure 9: Effects of resistance to communication campaigns and of the selective influence of neighbours

ulation and favour the adoption of a vegetarian diet. The way arguments build public opinion and may lead to behavioural changes can be simulated by the ABM, whereas this dynamic cannot be captured by the argumentation system alone. A comparison of the argumentation and ABM representations that emerged from this study is proposed in Table 2.

The simulation results and, more specifically, sensitivity analysis, showed that arguments in favour of meat reduction can be widely diffused in the population and that the repeatability of this result is dependent on several parameters. Among them, the ratio between the frequencies of crises and communication campaigns plays an important part in the stability of the results obtained over the simulations (Figure 6). It is interesting to notice that the initial proportion of vegetarians has little effect compared to that of the radius of crisis impact (Figure 7). Moreover, another parameter was demonstrated to impact the stability of the model, namely the scalability of the resistance rate. Indeed, when resistance to change increases in the case of food diet change, the results in terms of the final number of vegetarians are much more homogeneous over the simulations (Figure 8). This observation is confirmed in the particular cases where this enhanced resistance affects consumer receptivity to communication campaigns and differentlythinking neighbours (Figure 9).

A crucial issue in argument modelling and simulation is the completeness and the robustness of the information collected. Of course, such a complex issue could never pretend to be exhaustive. So how can the question of completeness be dealt with? A first possible direction is to combine different types of information, in particular quantitative sources of information such as large surveys which allow analyzing the repres- 
entativeness of different viewpoints, and qualitative ones which allow the identification of nuances. In this perspective, ongoing research extends the set of arguments elicited so as to include, on the one hand, quantitative results from a survey of 2,000 people questioned on their current and ideally intended food habits, and data from the French National Institute of Statistics and Economic Studies (INSEE) to model social networks at the level of a city; and on the other hand, qualitative results from 20 biographic interviews that provide in-depth understanding of the motivations, initiating events, brakes and facilitators of food diet changes on the long-term. A second possible direction is to observe the evolution of the plus-value brought by new information sources in time. When no more substantial novelty is brought, a fixed point is reached.

Another thorny issue is the perceived reliability of arguments and its role in opinion dynamics. Indeed, the way arguments are processed can also be refined. Presently, argument perception depends on the criteria addressed by the argument and on the importance of these criteria for each citizen. Other factors can impact the perception of an argument, in particular, the source of the argument (Pornpitakpan, 2004). Hence, messages from dubious sources can differentially impact citizens' responsiveness. Various types of influencing agents can be distinguished, such as lobbies, the government, companies, etc., with various strategies to diffuse arguments.

\section{Conclusions}

The method presented here formalizes arguments and attacks around vegetarian diets using an abstract argumentation approach. The argument network revealed the foremost importance of health issues surrounding vegetarian diets. The centrality of some of the arguments in the network allowed for identification of potential key arguments and/or controversies. The importance of health arguments in relation to ethical arguments should be further researched.

Argument networks take advantage of being used together with ABM to explore the emergent establishment of new social norms in the case of vegetarianism. The overall research demonstrates the potential of developing an ABM to predict the triggers impacting the dynamics of food habit changes. Crises - sanitary, environmental and ethical - lead to the adoption of vegetarian products by consumers. The results indicated that there is the potential of extending the reach of nutrition transition by acting on several parameters: counterbalance of advertising campaigns by awareness campaigns, wide diffusion of awareness messages, and measures encouraging people to maintain their new habits and deterring them from backing down.

An interesting issue to further investigate is how public opinion is formed in the case of crises, including situations where the assumptions that the whole population is well informed and the news is reliable do not hold.

\section{Acknowledgements}

The authors are grateful to the organizers of the MISS-ABMS 2017 International Summer School, for the support of the INRA "VITAMIN" project, and for the networking support of the COST Action CA15118 (FoodMC) project.

\section{References}

Amgoud, L. \& Prade, H. (2009). Using arguments for making and explaining decisions. Artificial Intelligence, 173(3-4), 413-436. doi:10.1016/j.artint.2008.11.006

An, L. (2012). Modeling human decisions in coupled human and natural systems: Review of agent-based models. Ecological Modelling, 229(SI), 25-36. doi:10.1016/j. ecolmodel.2011.07.010

Baroni, P. \& Giacomin, M. (2009). Semantics of abstract argument systems. (pp. 25-44). doi:10.1007/978-0-387-98197-0_2

Beardsworth, A. D. \& Keil, E. T. (1991). Vegetarianism, veganism, and meat avoidance: Recent trends and findings. British Food Journal, 93, 19-24. doi:10 . 1108/ 00070709110135231

Berger, T. (2001). Agent-based spatial models applied to agriculture: A simulation tool for technology diffusion, resource use changes and policy analysis. Agricultural Econom- 
ics, 25(2-3), 245-260. doi:10.1111/j.15740862.2001.tb00205.x

Besnard, P. \& Hunter, A. (2008). Elements of argumentation. MIT press Cambridge.

Bourguet, J.-R., Thomopoulos, R., Mugnier, M.-L. \& Abecassis, J. (2013). An artificial intelligence-based approach to deal with argumentation applied to food quality in a public health policy. Expert Systems with Applications, 40(11), 4539-4546. doi:10 . 1016/j.eswa.2013.01.059

Castella, J. C., Trung, T. N. \& Boissau, S. (2005). Participatory simulation of landuse changes in the northern mountains of vietnam: The combined use of an agentbased model, a role-playing game, and a geographic information system. Ecology and Society, 10(1).

Craig, W. J. \& Mangels, A. R. (2009). Position of the american dietetic association: Vegetarian diets. Journal of the American Dietetic Association, 109(7), 1266-1282. doi:10.1016/j.jada.2009.05.027

Deffuant, G., Amblard, F., Weisbuch, G. \& Faure, T. (2002). How can extremism prevail? a study based on the relative agreement interaction model. J. Artificial Societies and Social Simulation, 5.

Dung, P. M. (1995). On the acceptability of arguments and its fundamental role in nonmonotonic reasoning, logic programming and n-person games. Artificial Intelligence, 77(2), 321-357. doi:10.1016/0004-3702(94) 00041-X

Epstein, J. \& Axtell, R. (1996). Growing artificial societies: Social science from the bottom up. doi: $10.2307 / 20048043$

Filatova, T., Verburg, P. H., Parker, D. C. \& Stannard, C. A. (2013). Spatial agentbased models for socio-ecological systems: Challenges and prospects. Environmental Modelling \&5 Software, 45, 1-7. doi:10 . 1016/j.envsoft.2013.03.017

Francione, G. L. (2015). Animal rights: The abolitionist approach. Exempla Press.

Grimm, V. (1999). Ten years of individualbased modelling in ecology: What have we learned and what could we learn in the future? Ecological Modelling, 115(2-3), 129148. doi:10.1016/S0304-3800(98)00188-4
Jabs, J., Devine, C. M. \& Sobal, J. (1998). Model of the process of adopting vegetarian diets: Health vegetarians and ethical vegetarians. Journal of Nutrition Education, 30(4), 196-202. doi:10.1016/S00223182(98)70319-X

Janssen, M. A., Walker, B. H., Langridge, J. \& Abel, N. (2000). An adaptive agent model for analysing co-evolution of management and policies in a complex rangeland system. Ecological Modelling, 131(2-3), 249268. doi:10.1016/S0304-3800(00)00256-8

Janssen, M. A. \& Ostrom, E. (2006). Empirically based, agent-based models. Ecology and Society, 11 (2).

Matthews, R. B., Gilbert, N. G., Roach, A., Polhill, J. G. \& Gotts, N. M. (2007). Agentbased land-use models: A review of applications. Landscape Ecology, 22(10), 14471459. doi:10.1007/s10980-007-9135-1

McDonald, B. (2000). "once you know something, you can\&apos;t not know it" an empirical look at becoming vegan. Society and Animals, 8, 1-23. doi:10.1163 / $156853000 X 00011$

Ouerdane, W., Maudet, N. \& Tsoukiàs, A. (2010). Argumentation theory and decision aiding. Trends in Multiple Criteria Decision Analysis, 142. doi:10.1007/978-14419-5904-1_7

Parker, D. C., Manson, S. M., Janssen, M. A., Hoffmann, M. J. \& Deadman, P. (2003). Multi-agent systems for the simulation of land-use and land-cover change: A review. Annals of the Association of American Geographers, 93(2), 314-337. doi:10.1111/ 1467-8306.9302004

Peltonen, R., Nenonen, M., Helve, T., Hänninen, O., Toivanen, P. \& Eerola, E. (1997). Faecal microbial flora and disease activity in rheumatoid arthritis during a vegan diet. Rheumatology, 36(1), 64-68. doi:10.1093/ rheumatology/36.1.64

Popkin, B. M. (1993). Nutritional patterns and transitions. Population and Development Review, 19(1), 138-157. doi:10 . 2307 / 2938388

Pornpitakpan, C. (2004). The persuasiveness of source credibility: A critical review of five decades' evidence. Journal of Applied So- 
cial Psychology, 34(2), 243-281. doi:10. 1111/j.1559-1816.2004.tb02547.x

Rahwan, I. \& Simari, G. (2009). Argumentation in artificial intelligence. doi:10.1007/9780-387-98197-0

Railsback, S. F., Lytinen, S. L. \& Jackson, S. K. (2006). Agent-based simulation platforms: Review and development recommendations. Simulation-transactions of the Society for Modeling and Simulation International, 82(9), 609-623. doi:10.1177/ 0037549706073695

Rammel, C., Stagl, S. \& Wilfing, H. (2007). Managing complex adaptive systems - a co-evolutionary perspective on natural resource management. Ecological Economics, 63(1), 9-21. doi:10.1016/j.ecolecon.2006. 12.014

Robinson, D., Brown, D., Parker, D., Schreinemachers, P., Janssen, M., Huigen, M., ... Barnaud, C. (2007). Comparison of empirical methods for building agent-based models in land use science. Journal of Land Use Science, 2, 31-55. doi:10.1080/17474230701201349

Ruby, M. B. (2012). Vegetarianism. a blossoming field of study. Appetite, 58(1), 141-150. doi:10.1016/j.appet.2011.09.019

Samuelson, D. (2000). Designing organizations. OR/MS Today, 27, 6. Retrieved from https: / / www . informs . org / ORMS - Today / Archived - Issues / 2000 / orms - 12 - 00 / Designing-Organizations

Samuelson, D. A. \& Macal, C. M. (2006). Agentbased simulation comes of age. OR MS TODAY Institute for Operations Research and the Management, 33(4), 34.

Schelling, T. C. (1971). Dynamic models of segregation. Journal of Mathematical Sociology, 1(2), 143-186. doi:10 . 1080/ 0022250X.1971.9989794

Schluter, M., Baeza, A., Dressler, G., Frank, K., Groeneveld, J., Jager, W., ... Wijermans, N. (2017). A framework for mapping and comparing behavioural theories in models of social-ecological systems. Ecological Economics, 131, 21-35. doi:10.1016/j.ecolecon. 2016.08 .008
Simon, H. A. (1997). Models of bounded rationality: Empirically grounded economic reason. MIT press.

Taillandier, P., Gaudou, B., Grignard, A., Quang Nghi, H., Marilleau, N., Caillou, P., ... Drogoul, A. (2018). Building, composing and experimenting complex spatial models with the gama platform. GeoInformatica. doi:10.1007/s10707-018-00339-6

Thomopoulos, R. (2018). A practical application approach to argumentation for multicriteria analysis and decision support. Euro Journal on Decision Processes, 6(3-4, SI), 237-255. doi:10.1007/s40070-018-0087-2

Thomopoulos, R., Croitoru, M. \& Tamani, N. (2015). Decision support for agrifood chains: A reverse engineering argumentation-based approach. Ecological Informatics, 26(2, SI), 182-191. doi:10.1016/j.ecoinf.2014.05.010

Thomopoulos, R., Moulin, B. \& Bedoussac, L. (2017). Combined argumentation and simulation to support decision: Example to assess the attractiveness of a change in agriculture. (pp. 275-281).

Tilman, D. \& Clark, M. (2014). Global diets link environmental sustainability and human health. Nature, (515), 518-522. doi:10. 1038/nature13959

Verburg, P. \& Veldkamp, A. (2005). Introduction to the special issue on spatial modeling to explore land use dynamics. International Journal of Geographical Information Science, 19, 99-102. doi:10.1080/ 13658810410001713362

Vranken, L., Avermaete, T., Petalios, D. \& Mathijs, E. (2014). Curbing global meat consumption: Emerging evidence of a second nutrition transition. Environmental Science \& Policy, 39, 95-106. doi:10.1016/ j.envsci.2014.02.009

Walton, D. \& Macagno, F. (2015). A classification system for argumentation schemes. Argument \& Computation, 6(3), 219-245.

Xie, J., Sreenivasan, S., Korniss, G., Zhang, W., Lim, C. \& Szymanski, B. K. (2011). Social consensus through the influence of committed minorities. Physical Review E, $84(1,1)$. doi:10.1103/PhysRevE.84.011130 